\title{
Superiority of half-wavelength helicon antennae
}

\author{
L Porte, S M Yun, D Arnush ${ }^{1}$ and F F Chen
}

Electrical Engineering Department, University of California, Los Angeles, CA 90095-1594, USA

E-mail: laurie.porte@epfl.ch,seokmin.yun@lamrc.com and ffchen@ee.ucla.edu

Received 27 November 2002, in final form 6 January 2003

Published 2 May 2003

Online at stacks.iop.org/PSST/12/287

\begin{abstract}
Plasma densities produced by half- and full-wavelength (HW and FW)

helical antennae in helicon discharges are compared. It is found that HW

antennae are more efficient than FW ones in producing plasma downstream

from the antenna. The measured wave amplitudes and the apparent

importance of downstream ionization do not agree with computations.
\end{abstract}

\section{Introduction}

Helicon discharges have been studied intensively because they produce high density plasmas efficiently for use in materials processing, space propulsion, and basic plasma experiments. The most common antenna used to excite helicon waves is the Nagoya Type III antenna [1], a modification of which is the double-saddle coil of Boswell [2]. Helical antennae were first used by Shoji [3] and have been adopted by other workers [4]. Other types include single-loop antennae [5-7], doubleloop antennae [8], double half-turn antennae [9], quadrupole antennae [10], solenoid antennae [11], and bifilar rotating-field antennae [12]. Relative efficiencies of these designs have been compared by several groups [12-15] and in general the results agree with calculations [16-19]. In this paper, we compare helical antennae of different lengths and find surprising results that appear to disagree with theory.

Helical antennae designed to launch right-hand (RH) circularly polarized (azimuthal mode number $m=+1$ ) helicon waves have been found to be more efficient than those of opposite helicity $(m=-1)$ and also better than straight $(m= \pm 1)$ Nagoya Type III antennae. In an attempt to optimize RH helical antennae, we compared the standard halfwavelength $(\mathrm{HW})$ antenna with a full-wavelength $(\mathrm{FW})$ one, expecting that the FW antenna would be more efficient, since it would have a narrower $k$-spectrum and hence could be tuned to match the maximum plasma response. Surprisingly, we found that the opposite was true. The initial measurements were made by Porte in 1997. To be sure the results were valid, the experiment was repeated two years later by Yun, who found essentially identical results and extended the work by studying the $B_{0}$ dependence. In the meantime, the HELIC code [20]

1 Deceased 25 April 2003. was developed and improved to give theoretical insight into the behaviour of different antennae. However, the issue could not be resolved with this tool, indicating that the behaviour of helicon discharges still contains an unknown physical mechanism.

\section{Apparatus}

Experiments were carried out in the long tube shown in figure 1. The field coils provided a uniform $B_{0}$ up to $1 \mathrm{kG}$; the gas feed was near the midplane; and the antenna was near one end of the machine, as shown. Unless otherwise specified, the discharge had a fill pressure $p$ of 20 mTorr of argon, with $1.4 \mathrm{~kW}$ of power $P_{\text {rf }}$ at $27.12 \mathrm{MHz}$ and an $800 \mathrm{G}$ field $B_{0}$. Density $n$, electron temperature $K T_{\mathrm{e}}$, and space potential $V_{\mathrm{s}}$ were measured with RF-compensated Langmuir probes [21]: a dogleg probe for axial scans and probes in two ports for radial scans. Measurements of the wave field components $B_{z}$ and $B_{\theta}$ were made with similarly mounted B-dot probes for radial and axial scans. The data were reproducible after venting the machine and subsequent pumpdown, even after long periods of inactivity.

Two antennae were compared: a $10 \mathrm{~cm}$ long HW antenna (HW10) of $20 \mathrm{~cm}$ wavelength, and a $20 \mathrm{~cm}$ long FW antenna (FW20) of the same helicity. The HW10 antenna had been found to give the highest densities and had been adopted as the standard. Later, a $15 \mathrm{~cm}$ FW antenna (FW15) was also tested, since HELIC computations showed that it gave somewhat higher plasma loading resistance than the $20 \mathrm{~cm}$ one. The antennae were constructed of $1 \mathrm{~cm}$ wide copper strap. Water cooling of the antennae and the $B_{0}$ coils was obviated by operating $B_{0}$ in $\sim 0.5 \mathrm{~s}$ pulses, and the RF in $\sim 10 \mathrm{~ms}$ pulses. Probe measurements were made during the flat top of the RF 


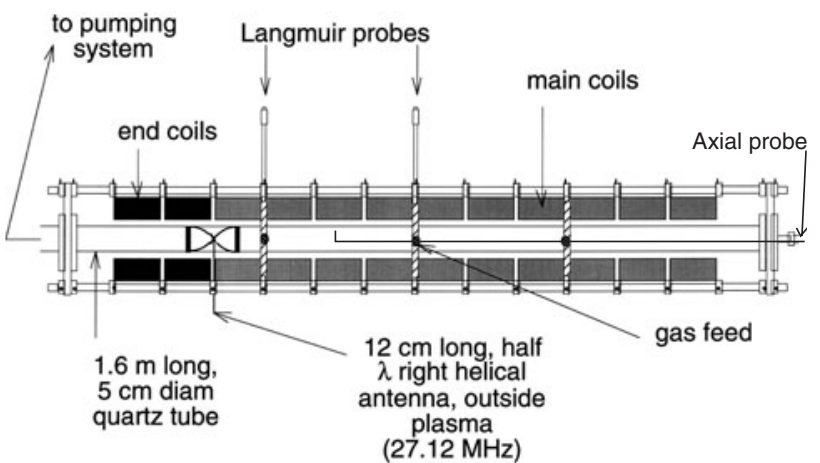

Figure 1. Diagram of the apparatus.

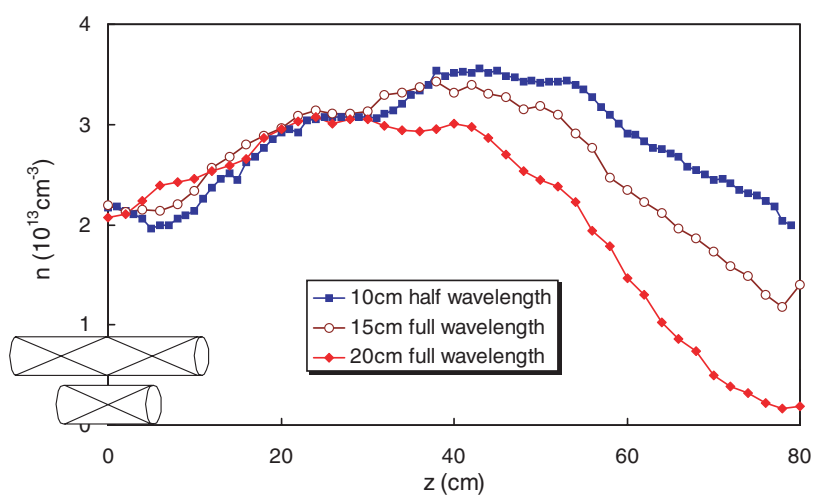

Figure 2. Axial variation of density produced by the HW10 ( $\square$ ), FW15 (O), and FW20 ( ) antennae. The lengths and locations of the antennae are shown at the lower left corner.

pulse, and the RF matchbox was tuned for $<1 \%$ reflection before each measurement.

\section{Data, series 1}

Figure 2 shows the axial density profiles obtained with the three antennae under otherwise identical conditions. The HW10 antenna produces much higher downstream density than the FW20 antenna, which is essentially two HW10 antennae laid end-to-end. Note, however, that under the antennae, and in the near-field, the FW20 antenna is superior, as expected a priori. The fact that $n(z)$ peaks $\sim 50 \mathrm{~cm}$ downstream from the antenna was attributed [22] to pressure balance, followed by radial diffusion loss. That is, as $K T_{\mathrm{e}}$ decays away from the antenna, $n$ must rise to keep $n K T_{\mathrm{e}}$ constant. The force $e E_{z}$ modifies this condition slightly. Other factors affecting the position of the density peak are ion flow produced at the antenna and downstream ionization. These effects will be discussed later, but they would not be expected to cause a large difference in total ionization when only the length of the antenna is changed. The positions of the density peaks of the three antennae and the downstream densities were entirely reproducible over several months and machine vents and pumpdowns. The densities nearer the antenna were not exactly reproducible but had the same qualitative behaviour ${ }^{2}$. That the HW10 antenna should create more downstream plasma

2 Data supporting these assertions can be found in the report LTP-110 (October 2001), accessible from www.ee.ucla.edu/ ltptl

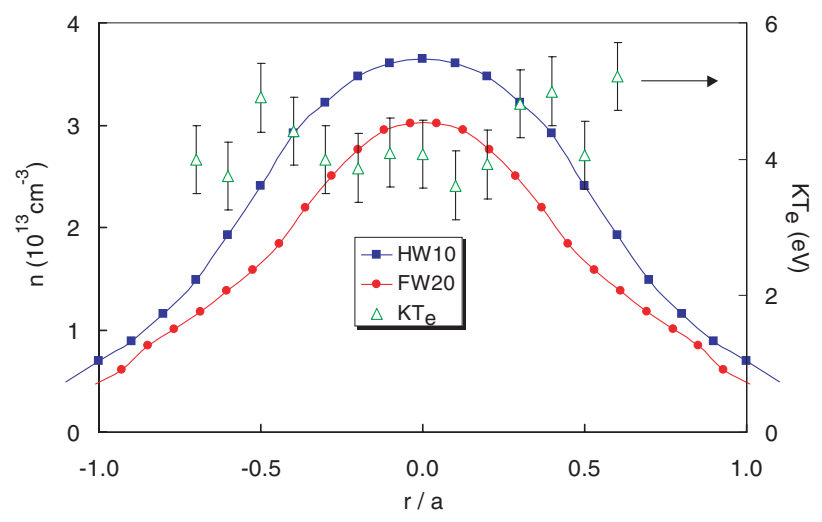

Figure 3. Radial density profiles $26 \mathrm{~cm}$ downstream from antenna midplane for the $10 \mathrm{~cm} \mathrm{HW} \mathrm{( \square )} \mathrm{and} 20 \mathrm{~cm} \mathrm{FW} \mathrm{( \bullet )} \mathrm{antennae.} \mathrm{Data}$ from both sides of the axis have been averaged to produce symmetric curves. The electron temperature $(\triangle)$ was measured with the HW antenna.

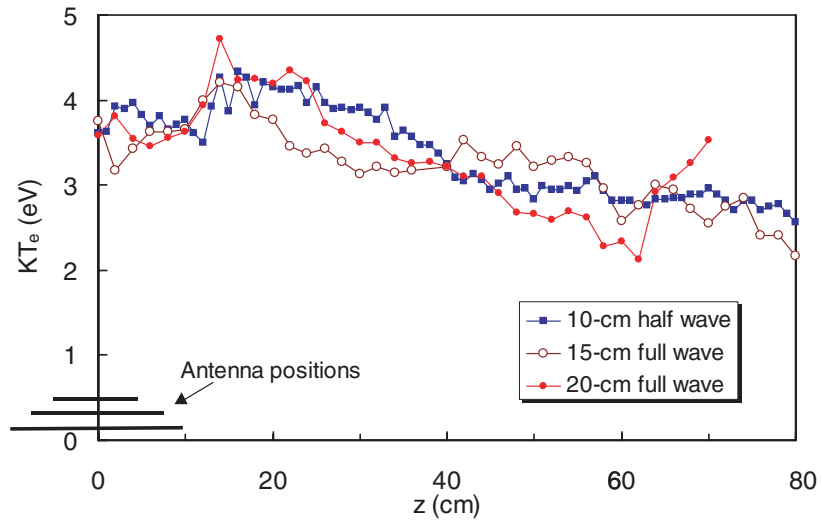

Figure 4. Axial variation of electron temperature with the three antennae. The rise in $K T_{\mathrm{e}}$ for the FW20 antenna at large $z$ is caused by failure of RF compensation at low densities.

than the FW20 antenna was unexpected. Several checks were made to confirm that both antennae produced normal helicon discharges. For instance, figure 3 shows that the radial density profiles with the HW10 and FW20 antennae are similar and quite normal. The electron temperature is seen to be about $4 \mathrm{eV}$. The wave fields were also found to have the usual Bessel function profiles ${ }^{2}$.

To see if the plasma conditions differed along the axis for the three cases, other parameters were measured. Figure 4 shows the variation of $K T_{\mathrm{e}}$ at various positions along $z$. Some differences among the antennae can be seen, but the behaviour is not consistent with the density behaviour in figure 2. For instance, $K T_{\mathrm{e}}$ for the FW20 antenna falls faster than the others, but there is no corresponding rapid rise in density as pressure balance would require. In both the upstream and downstream regions the differences in $K T_{\mathrm{e}}$, and hence the local ionization rates, do not correspond to the measured densities in figure 2 . Figure 5 shows the wave amplitude versus $z$. The characteristic beating [23] of various radial modes with different $k$ is seen, and the major peaks are in approximate agreement for all cases. The phase of the dominant spectral component versus $z$ was measured $^{2}$, and from this the local wavelength $\lambda$ was derived 
and is shown in figure 6 . It is seen that, in the first $40 \mathrm{~cm}, \lambda$ decreases as $n$ rises, in agreement with the helicon dispersion relation. However, all three antennae excite essentially the same waves. None of these measurements yields a clue as to the why the HW10 antenna produces more plasma. Figure 7 shows radial density profiles at two axial positions. Near the antenna, $n(r)$ is sharply peaked; further downstream, radial

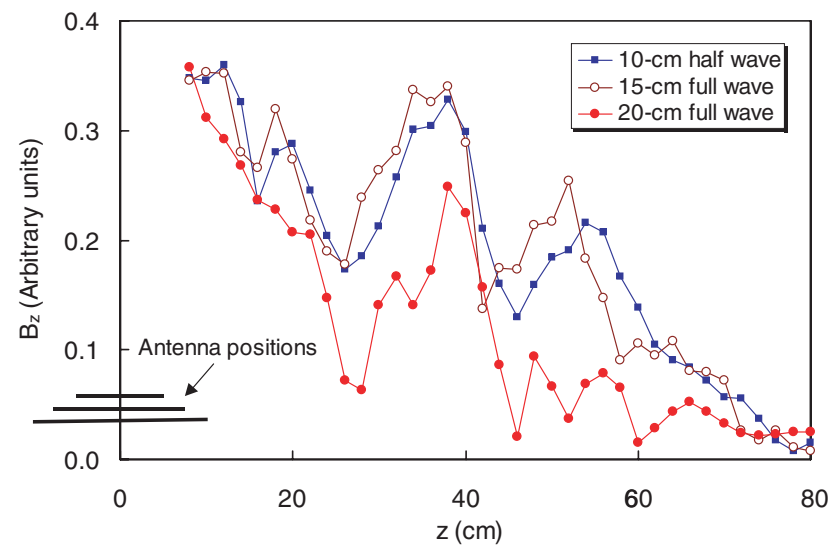

Figure 5. Wave amplitude $\left|B_{z}\right|$ versus $z$ excited by the three antennae.

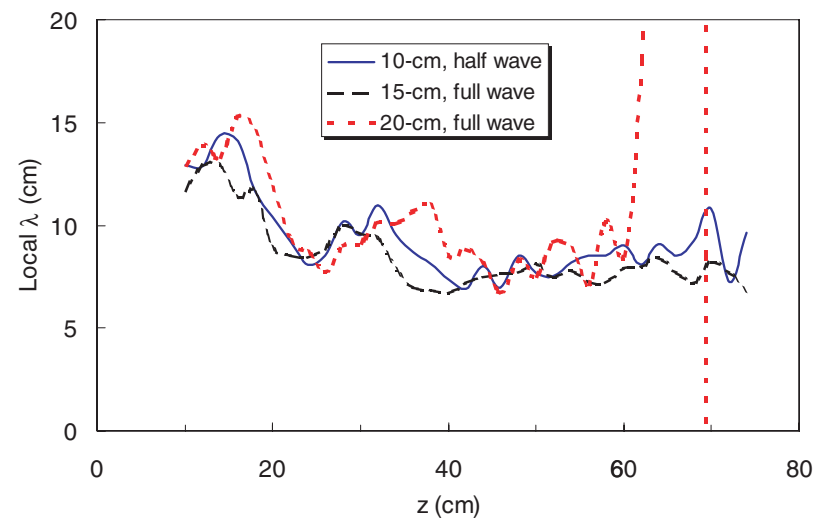

Figure 6. Local wavelength versus $z$ for the three antennae. The anomaly at $z>60 \mathrm{~cm}$ for the FW20 antenna is caused by the weak signal there.

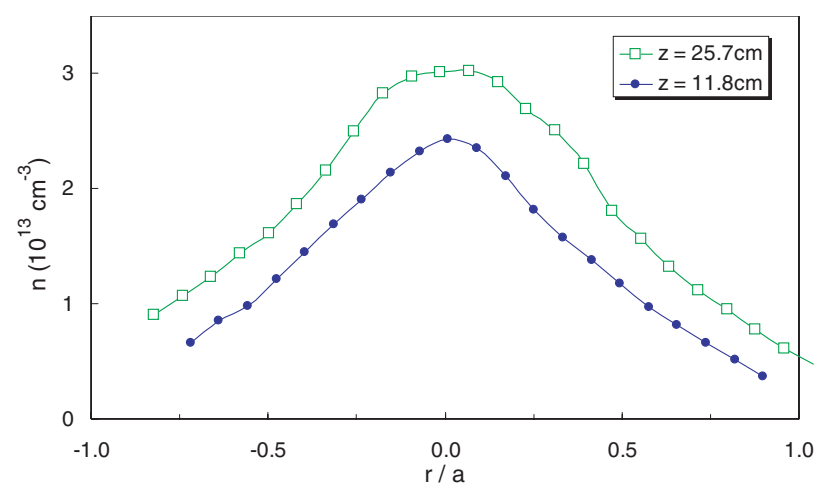

Figure 7. Radial density profiles at two axial positions, taken with the FW20 antenna. diffusion causes it to have a more parabolic shape. Since $n(0)$ is shown in figure 3 , the broadening of the profile means that not only is the downstream density highest for the HW10 antenna, but the volume integrated density is also highest.

\section{Computations}

Response of the plasma to excitation by various antennae was computed with the HELIC code of Arnush [20]. Aside from its user-friendly interface, this code is similar to those used by numerous other helicon groups [24-29], and its results should be reproducible by any of these other codes as long as nonuniform $n(r)$ profiles, TG modes, and collisional damping are included. These codes are based on the cold-plasma dielectric and usually assume axially uniform equilibrium conditions, though end boundaries can be included. Though the loading resistance can be computed this way, the equilibrium density cannot be found without considering plasma ionization and transport. Only recently have attempts [30-32] been made to couple the helicon equations with transport and continuity equations to give the density profiles.

For design purposes, the plasma loading was computed with HELIC for uniform $n(r)$. Figure 8(a) compares the three antennae in regard to the power absorbed into various plasma modes. The FW15 antenna was chosen because its absorption spectrum in the two main peaks is similar to that
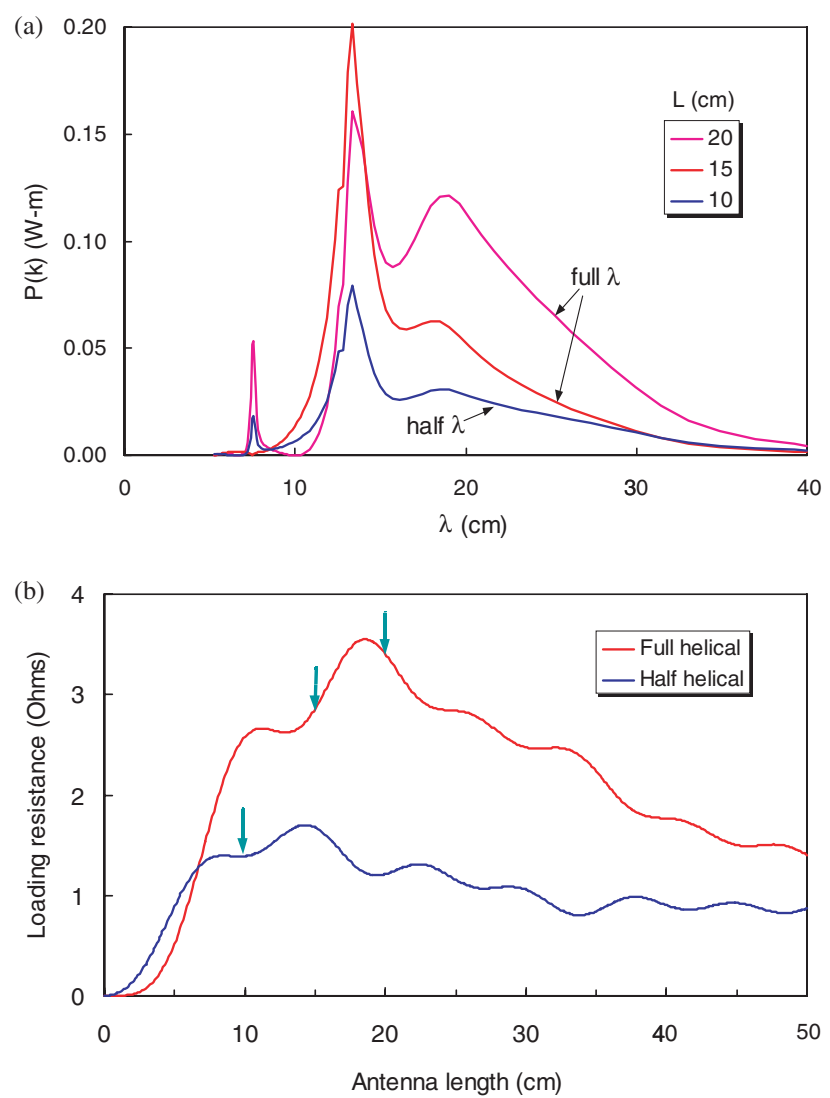

Figure 8. (a) RF absorption per unit $k$ versus helicon wavelength for the FW20, FW15, and HW10 antennae. (b) Total plasma loading versus antenna length for FW (top curve) and HW antennae. The three antennae used are marked with arrows. 


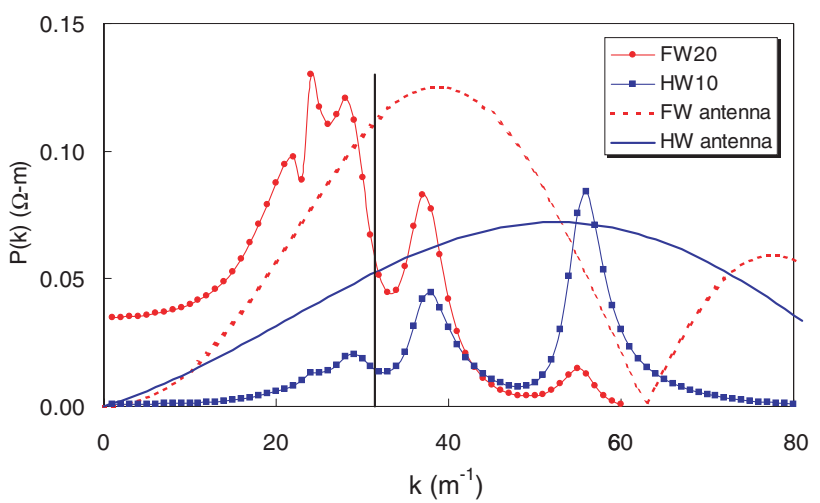

Figure 9. Computed $k$-spectra of energy deposition by the FW20 (0) and HW10 ( $\square$ ) antennae under the experimental conditions. The lines are the $k$-spectra of the antennae in vacuum. The vertical line marks the $k$ of the antenna winding.

of the HW10 antenna. The total loading resistance shown in figure $8(b)$ is much higher for both $\mathrm{FW}$ antennae than for the HW10 antenna. In the downstream region, this ordering is reversed in the experimental data, as if the total length of the antenna is more important than matching its helicity with the dominant helicon wavelength.

In the following HELIC calculations the radial density profile was taken into account with an analytic fit to the curves in figure 3 , and the other parameters were $B_{0}=800 \mathrm{G}$, $p=20 \mathrm{mTorr}, K T_{\mathrm{e}}=4 \mathrm{eV}, f=27.12 \mathrm{MHz}$, and $n_{\text {peak }}=3.6 \times 10^{13} \mathrm{~cm}^{-3}$. The $k$-spectrum of energy absorbed by the plasma at various wavelengths is compared between the HW10 and FW20 antennae in figure 9. This is a superposition of the plasma response and the $k$-spectrum of the antenna, shown by the respective curves. In spite of the fact that the FW20 antenna does not match the plasma resonances as well as does the HW10 antenna, the total loading is much higher for the FW20. Note that neither antenna has its peak response matching the wavelength of the coil, whose $k$ value is marked by the vertical line. Apparently, the end rings have an appreciable effect on the antenna spectrum. The two antennaes' end rings differ not only by their separation, but also in the relative direction of the currents in them [12]. Surprisingly, the $P(k)$ spectra of FW and HW antennae of the same length are similar in shape, with the FW spectrum higher; the pitch of the winding does not appear to matter.

Axial power deposition profiles for the three antennae are shown in figure 10. The current in each antenna was assumed to be 1A; the FW15 antenna was the most efficient in this case. In the neighbourhood of $z=50 \mathrm{~cm}$, where the HW10 antenna gives a density peak, the absorption is $13 \%$ of the maximum, even when the decay in $K T_{\mathrm{e}}$ has been neglected. Therefore, downstream ionization is not predicted to contribute to the dominance of the HW10 antenna in that region.

We have also computed the radial absorption profile $P(r)$. It was thought that perhaps most of the FW power was deposited near the edge, where the plasma created is more easily lost than if created near the axis. However, it is found that, if anything, the FW antenna had more central deposition ${ }^{2}$. Since the antenna windings have $m=1$ symmetry, they couple primarily to $m=1$ helicon waves. However, since the antenna has finite length, coupling to other odd $m$-numbers is also

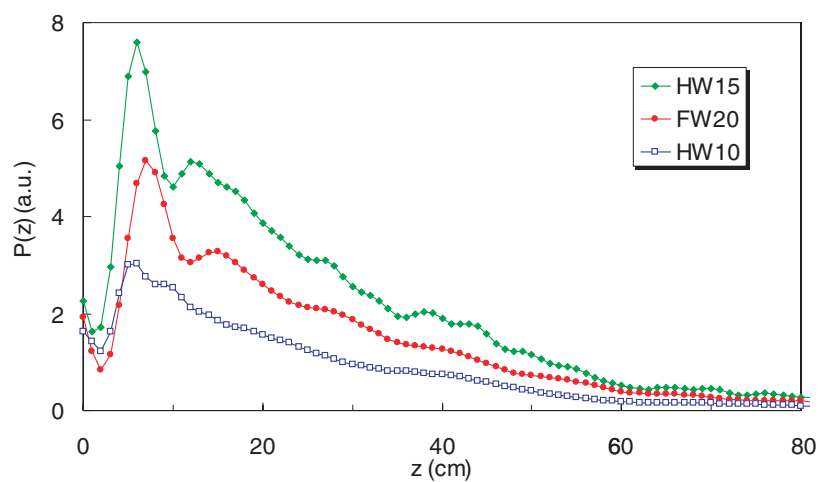

Figure 10. Calculated axial power deposition profiles for three antennae, integrated over the cross section. Conditions were: $B_{0}=800 \mathrm{G}, p=10 \mathrm{mTorr}$, and $K T_{\mathrm{e}}=4 \mathrm{eV}$, all uniform. The density was uniform along $z$ but had the experimental radial profile with $n_{\text {peak }}=3.6 \times 10^{13} \mathrm{~cm}^{-3}$.

possible. The loading by $m=3$ and 5 modes was computed, but it was found that $m>1$ adds negligibly to the total absorption.

Of the effects that are not included in the HELIC code, these are the most obvious: (1) axial gradients in $n$ and $K T_{\mathrm{e}}$ : as seen in figure 4 , there does not appear to be a difference in $T_{\mathrm{e}}(z)$ for the HW10 and FW20 antennae that could give higher downstream density for the former. (2) Ionization by non-Maxwellian electron populations: an ionizing electron of, say, $50 \mathrm{eV}$ would have a mean free path of $\sim 10 \mathrm{~cm}$, dominated by neutral collisions at $10 \mathrm{mTorr}$. It is not likely for these to reach the density peak at $z=50 \mathrm{~cm}$. However, if neutral depletion lowers the central pressure to $2 \mathrm{mTorr}, \mathrm{e}, \mathrm{g}$. then it would be possible for Landau damping of helicon waves to produce a few of such electrons. Attempts [33] to detect fast electrons in our laboratory have yielded an upper limit of $10^{-4}$ of the total density. In any case, the FW antenna, with its purer spectrum, should produce more of these electrons than the HW antenna. (3) Ion flow out of the antenna region: if there is little downstream ionization, ions leaving the antenna should be unidirectional. In that case, the Bohm criterion for monotonic sheath formation should obtain, and the ions must stream out with the ion acoustic velocity. This effect has been inferred previously [34]. The ion momentum then carries the plasma downstream, raising the density there over that in the static case. This effect would, however be the same for both HW and FW antennae. (4) Radial transport: HELIC is not a diffusion code, and the possibility of a difference in diffusion cannot be ruled out.

\section{Data, series 2}

The following measurements were made in the same apparatus two years later with a $5-\mathrm{cm}$ long HW antenna and a $10-\mathrm{cm}$ long FW antenna. The variation of $n$ with $B_{0}$ is shown in figure 11. Density jumps at critical fields, typical of helicon discharges, are seen with both antennae. Also seen is a small density peak near $50 \mathrm{G}$, another helicon characteristic. A third well-known effect is the much lower density produced when the antenna helicity is reversed to match the $m=-1$ mode. Figure 12 shows axial density profiles at different fields $B_{0}$. At high $B_{0}$, the dominance of the HW antenna shown in figure 2 


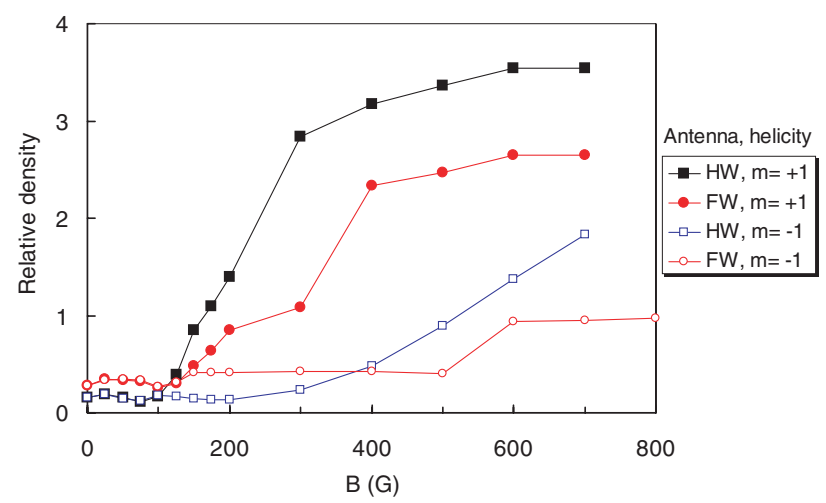

Figure 11. Density versus magnetic field at $r=2 \mathrm{~cm}, z=24 \mathrm{~cm}$ with HW5 and FW10 antennae at $857 \mathrm{~W}$ and 10 mTorr of Ar. The $m=-1$ mode is excited by reversing the direction of $B_{0}$.

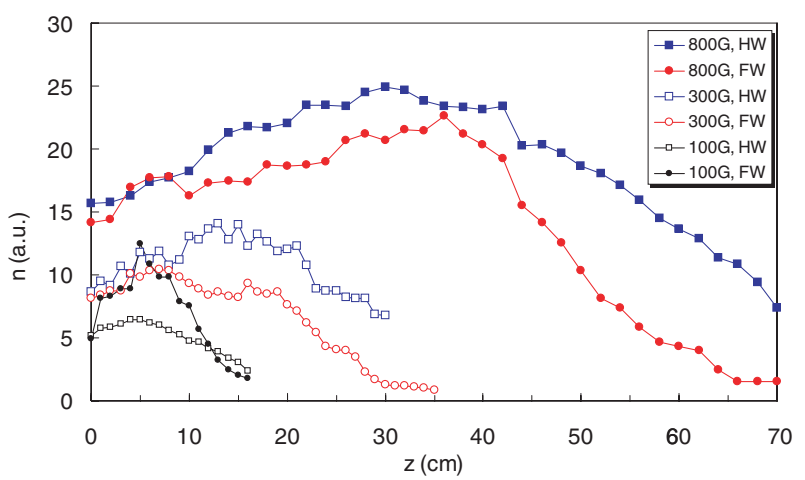

Figure 12. Axial density profiles with the HW5 $(\square, \mathbf{\square})$ and FW10 $(\mathrm{O}, \bullet)$ antennae at three magnetic fields.

is reproduced. At low $B_{0}$, below the density jumps, the FW antenna produces higher $n$. Radial density profiles are shown in figure 13 for six values of $B_{0}$. At $100 \mathrm{G}$ and below, both antennae are inefficient, but the FW antenna is somewhat better. The HW antenna causes a density jump before the FW one does (cf figure 11), and at $200 \mathrm{G}$ the HW antenna is superior. It continues to dominate up to the highest field of $800 \mathrm{G}$. Note that at fields beyond the density jump the profiles assume a 'triangular' shape, which has been explained by ion-electron dominated collisions together with a TG-mode absorption profile [35]. Thus, the superior performance of the HW antenna is manifest only at B-fields beyond the density jump.

The density data confirm the superiority of the HW antenna seen in the data of series 1 but do not provide an explanation. More light on the problem is provided by measurements of the antenna loading vs $B_{0}$. By measuring the voltage and current in the antenna and the phase between them, one obtains in figure 14 the resistance $R$ and reactance $|X|$ seen by the antennae. These are considerably higher for the FW antenna than for the HW antenna. The higher voltage on the FW antenna is expected because of its longer length, and hence higher inductance. Its higher loading resistance agrees with the HELIC results shown in section 4, but it should result in higher density, not lower. $R$ versus $P_{\mathrm{rf}}$ is shown in figure 15 , together with $n$. The HW antenna has its density jump at much lower power than does the FW antenna, presumably because its spectrum matches the plasma modes better (cf figure 9). Moreover, it yields higher density at all $P_{\mathrm{rf}}$,
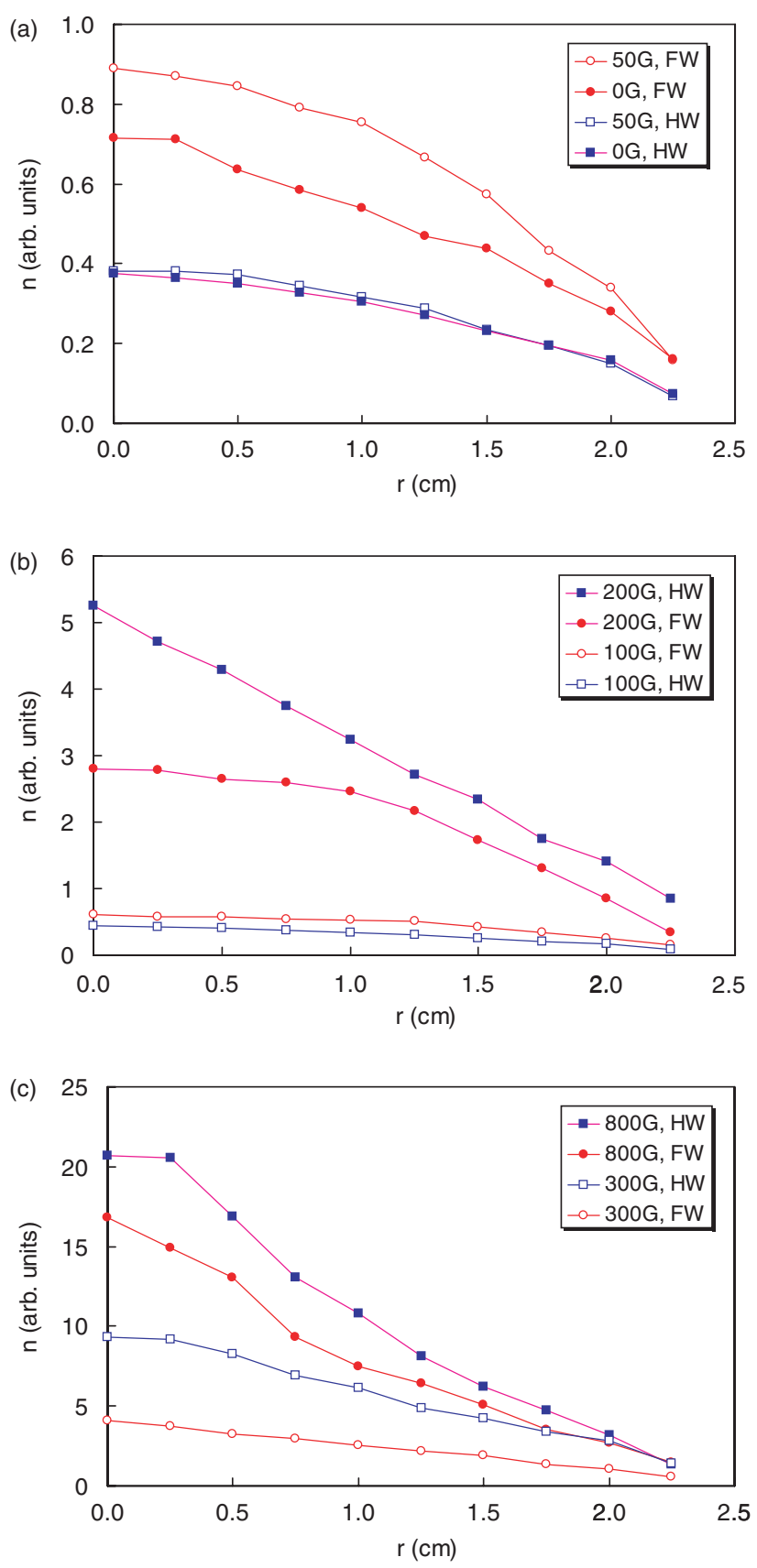

Figure 13. Radial density profiles at $z=24 \mathrm{~cm}$ at various $B_{0}$ with the HW $(\square, \boldsymbol{\square})$ and FW $(\mathrm{O}, \boldsymbol{\bullet})$ antennae.

even though $R$ is smaller at all $P_{\mathrm{rf}}$. Computed curves of $P(k)$ and $P(z)$ are similar to those in figures 9 and 10, except that the HW5/FW20 coupling ratio is expected to be even smaller than the HW10/FW20 ratio, contrary to the experimental results.

Figure 16 shows radial profiles of space potential for the two antennae. These profiles would be low in the centre if electrons were magnetically confined, and they would be peaked in the centre if the electrons obeyed the Boltzmann relation and followed the shape of the density profile. The latter occurs in short machines where electrons can cross field lines via the short-circuit effect at the endplate sheaths. The fact that $V_{\mathrm{s}}(r)$ is essentially flat in the body of the discharge means that the machine length achieves a balance between 


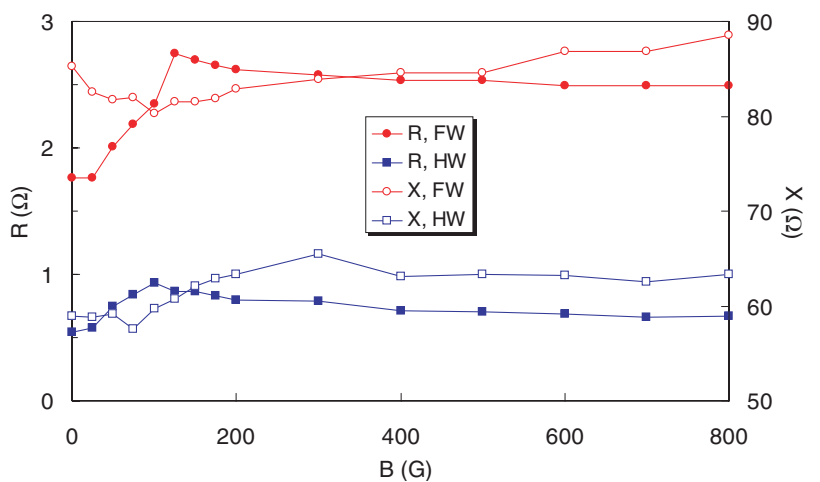

Figure 14. Magnitudes of loading resistance (solid points) and reactance (open points) presented by the plasma to the HW ( $\square$, and $\mathrm{FW}(\mathrm{O}, \bullet)$ antennae.

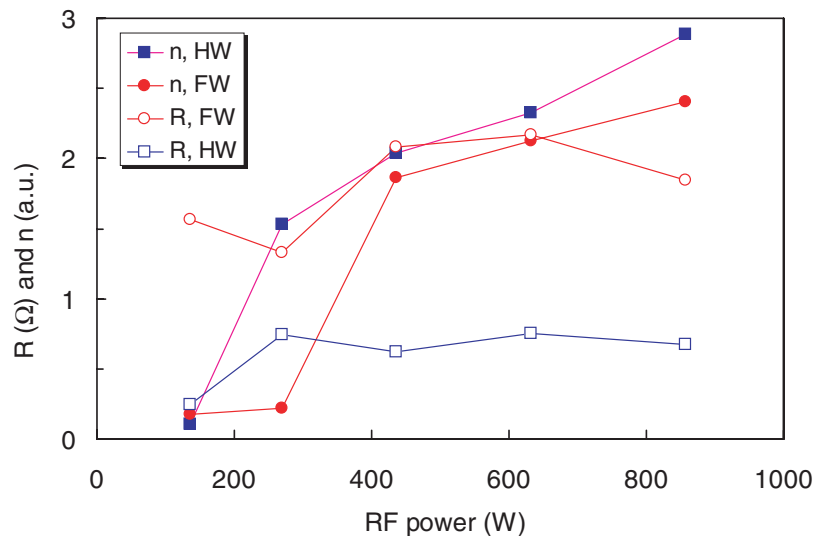

Figure 15. Density (solid points) and loading resistance (open points) versus RF power for the HW $(\square, \square)$ and $\mathrm{FW}(\mathrm{O}$, antennae.

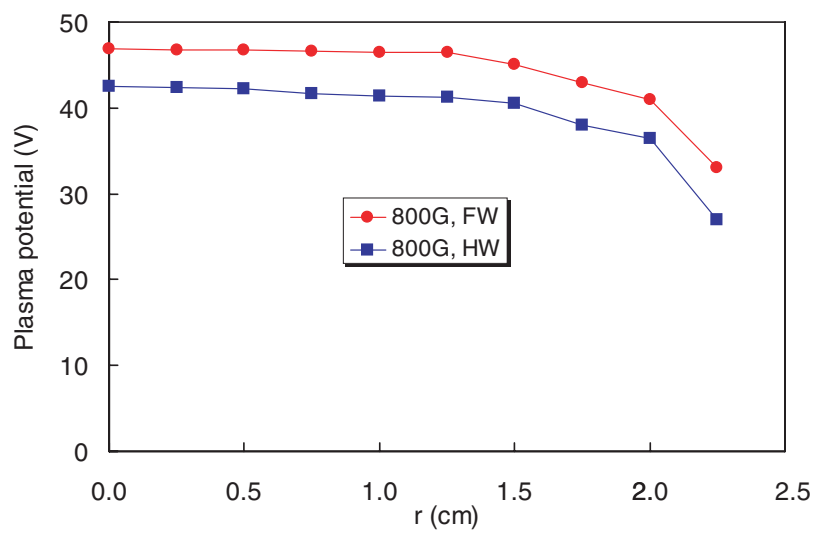

Figure 16. Plasma potential versus radius at $800 \mathrm{G}, z=48 \mathrm{~cm}$ for the FW (-) and HW (ם) antennae.

these two effects. The important point to notice in figure 16 is that $V_{\mathrm{s}}$ is higher for the FW antenna. This would cause ions to be lost radially faster than for the HW antenna, while electrons can follow the ions by the partial short-circuit effect. It is reasonable for $V_{\mathrm{s}}$ to be higher for the $\mathrm{FW}$ antenna because the applied voltage is higher, and capacitive coupling is more effective. This would cause large RF oscillations at the edge which the electrons can follow but the ions cannot, because of the high frequency. The $\theta$-component of the

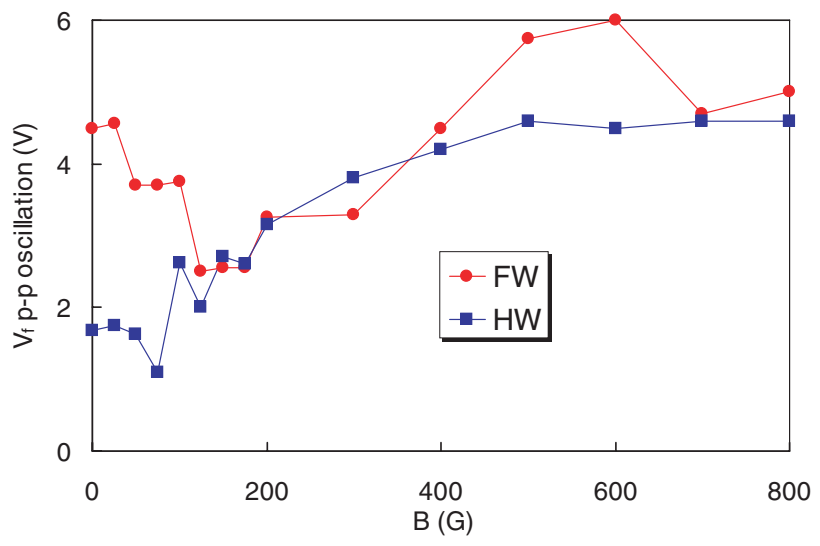

Figure 17. Peak-to-peak oscillation amplitude in floating potential vs $B_{0}$ at $z=24 \mathrm{~cm}, r=1.5 \mathrm{~cm}$ for the two antennae.

capacitive electric field would cause radial oscillations of the electron guiding centres, causing an enhanced electron loss at the edge, raising the plasma potential. Evidence of these oscillations is seen in figure 17, which shows peak-to-peak amplitudes of floating potential oscillations vs $B_{0}$. Below the critical field of $\sim 150 \mathrm{G}$ where the density jump occurs, capacitive coupling may be important, and the HW antenna causes larger oscillations because of its higher impedance. At high fields, there is some evidence that the HW antenna causes larger oscillations even though inductive coupling should be dominant. This raises the possibility that the FW antenna suffers from faster plasma loss due to anomalous diffusion, but this in only a conjecture at this point.

\section{Conclusions}

In two separate experiments, HW, RH helical antennae have been found to produce higher downstream density and more total ionization than FW antennae of the same helicity. Detailed calculations based on inductive coupling to heliconTG waves predict the opposite; namely, that rf absorption should be higher for FW than for HW antennae. To our knowledge, this effect has not previously been reported in the literature either experimentally or theoretically. It is particularly puzzling that the density difference occurs far downstream from the antenna. There is so far no explanation for this effect, though measurements suggest that FW antennae may cause faster radial transport via oscillations. This experiments show that there are fundamental mechanisms in the operation of helicon discharges that are still not understood, so that further studies on helicon physics are warranted.

\section{References}

[1] Okamura S et al 1986 Nucl. Fusion 261491

[2] Boswell R W 1984 Plasma Phys. Control. Fusion 261147

[3] Shoji T, Sakawa Y, Nakazawa S, Kadota K and Sato T 1993 Plasma Sources Sci. Technol. 25

[4] Kraemer M, Lorenz B and Clarenbach B 2002 Plasma Sources Sci. Technol. 11 A120

[5] Sakawa Y, Koshikawa N and Shoji T 1996 Appl. Phys. Lett. 69 1695

[6] Carter C and Khachan J 1999 Plasma Sources Sci. Technol. 8432 
[7] Shinohara S and Yonekura K 2000 Plasma Phys. Control Fusion 4241

[8] Tynan G R et al 1997 J. Vac. Sci. Technol. A 152885

[9] Degeling A W, Jung C O, Boswell R W and Ellingboe A R 1996 Phys. Plasmas 32788

[10] Kim J H, Yun S M and Chang H Y 1996 IEEE Trans. Plasma Sci. 241364

[11] Kim J H, Yun S M and Chang H Y 1996 Phys. Lett. A 22194

[12] Miljak D G and Chen F F 1998 Plasma Sources Sci. Technol. 761

[13] Chen F F 1992 J. Vac. Sci. Technol. A 101389

[14] Shinohara S and Soejima T 1998 Plasma Phys. Control. Fusion $\mathbf{4 0} 2081$

[15] Shinohara S and Shamrai K P 2000 Plasma Phys. Control Fusion $\mathbf{4 2} 865$

[16] Kamenski I V and Borg G G 1996 Phys. Plasmas 34396

[17] Kraemer M 1999 Phys. Plasmas 61052

[18] Schneider D A, Borg G G and Kamenski I V 1999 Phys. Plasmas 6703

[19] Shamrai K P and Pavlenko V P 1997 Phys. Scripta 55612

[20] Arnush D 2000 Phys. Plasmas 73042
[21] Sudit I D and Chen F F 1994 Plasma Sources Sci. Technol. 3162

[22] Sudit I D and Chen F F 1996 Plasma Sources Sci. Technol. 543

[23] Light M, Sudit I D, Chen F F and Arnush D 1995 Phys. Plasmas 24094

[24] Cho S and Kwak J G 1997 Phys. Plasmas 44167

[25] Kamenski I V and Borg G G 1998 Computer Phys. Commun. 11310

[26] Mouzouris Y and Scharer J E 1998 Phys. Plasmas 54253

[27] Enk Th and Kraemer M 2000 Phys. Plasmas 74308

[28] Park B H, Yoon N S and Choi D I 2001 IEEE Trans. Plasma Sci. 29502

[29] Shamrai K P and Shinohara S 2001 Phys. Plasmas 84659

[30] Carter M D, Baity F W Jr, Barber G C, Goulding R H, Mori R H, Sparks D O, White K F, Jaeger E F, Chang-Diaz F R and Squire J P 2002 Phys. Plasmas 95097

[31] Cho S W and Lieberman M A 2003 Phys. Plasmas 10882

[32] Bose D, Govindan T R and Meyyappan M 2003 IEEE Trans. Plasma Sci.

[33] Chen F F and Blackwell D D 1999 Phys. Rev. Lett. 822677

[34] Tynan G R et al 1997 J. Vac. Sci. Technol. A 152885

[35] Chen F F 1998 Plasma Sources Sci. Technol. 7458 